\title{
Point-to-origin experiments in VR revealed novel qualitative errors in visual path integration
}

\author{
Bernhard E. Riecke* \& Jan M. Wiener ${ }^{\dagger}$ \\ Max Planck Institute for Biological Cybernetics, Tübingen, Germany
}

Introduction Even in state-of-the-art virtual reality (VR) setups, participants often feel lost when navigating through virtual environments. In psychological experiments, such disorientation is often compensated for by extensive training and performance feedback. The current study investigated participants' sense of direction by means of a rapid point-to-origin task without any training or performance feedback. This allowed us to study participants' intuitive spatial orientation processes in VR while minimizing the influence of higher cognitive abilities and compensatory strategies. From an applied perspective, such a paradigm could be employed for evaluating the effectiveness and usability of a given VR setup for enabling natural and unencumbered spatial orientation even for firsttime users, which is important for tasks such as architecture walkthroughs, evacuation scenario training, or driving/flight simulators.

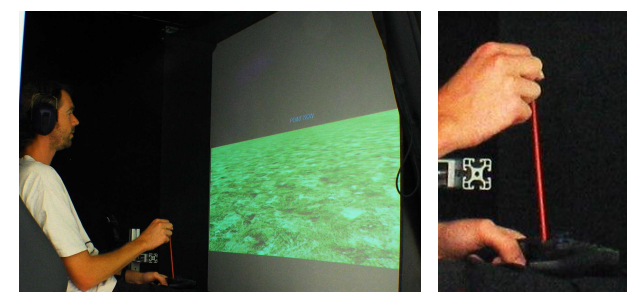

Figure 1: VR setup showing a participant with the pointing device (modified game-pad) seated in front of the projection screen displaying a textured ground plane devoid of any landmarks.

Methods \& Results After visually displayed passive excursions along one- or two-segment trajectories including a simulated rotation $\left(0^{\circ}, 30^{\circ}, 60^{\circ}, 90^{\circ}, 120^{\circ}, 150^{\circ}\right.$, or $\left.170^{\circ}\right), 16$ naive participants were asked to point back to the origin of locomotion "as accurately and quickly as possible" (Fig. 1). Despite using a high-quality video projection $\left(84^{\circ} \times 63^{\circ} \mathrm{FOV}, 1400 \times 1050\right.$ pixels resolution), participants' overall performance was rather poor and showed considerable systematic as well as variable errors (Fig. 2). Moreover, six of the 16 participants consistently exhibited striking qualitative errors (left-right errors) that have to our knowledge not been observed in comparable real world experiments. That is, those left-right inverters consistently pointed into the opposite (left-right

*e-mail: bernhard.riecke@tuebingen.mpg.de

†e-mail: jan.wiener@college-de-france.fr

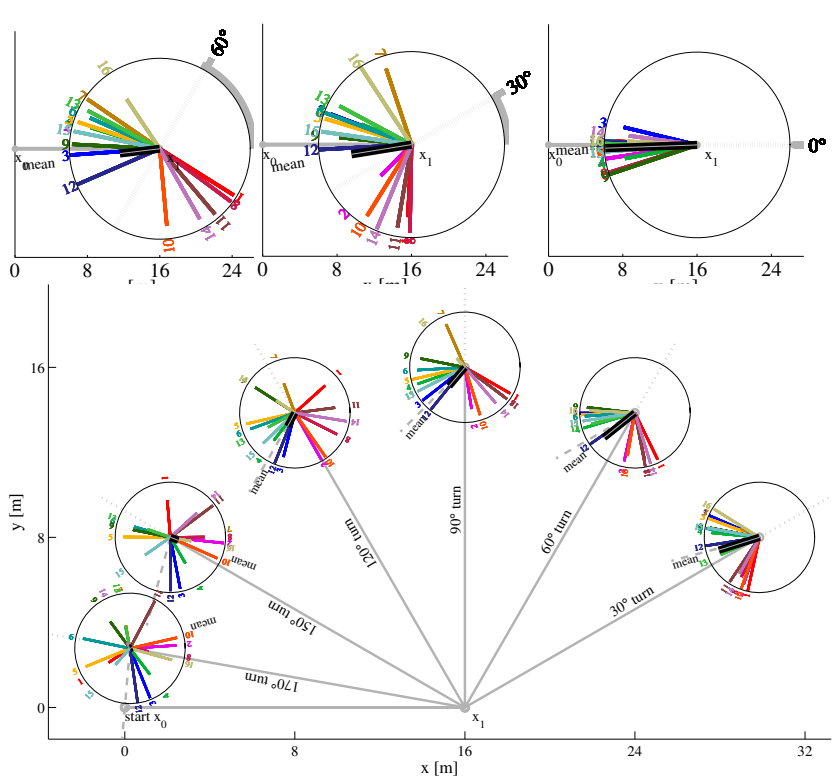

Figure 2: Top: Sample data for the 1-segment condition illustrating the systematic left-right errors for six of the 16 participants. Plotted is a top-down schematic view of the excursion path (in solid gray) from the start point $x_{0}$ to the endpoint $x_{1}$ and the subsequent turn by $60^{\circ}, 30^{\circ}$, or $0^{\circ}$. The circular mean pointing direction of each participant is indicated by the colored bars and subject IDs. The length of the mean pointing vector indicates the consistency of the individual pointing directions. Bottom: 2 -segment condition. Note the side errors for the six left-right inverters (depicted in reddish colors, same participants as in the 1-segment condition).

mirrored) direction despite showing a clear sensitivity to turning angles. When tested with actual walking in a pre-test, however, they did not show these left-right errors.

Discussion \& conclusions Data from [Klatzky et al. 1998] suggests that the left-right errors in the 2-segment conditions of our study might have been caused by a failure to update one's internal representation of heading. In the 1-segment conditions, however, the six participants showing consistent left-right inversions clearly did update their heading (albeit in the wrong direction, cf. Fig. 2) and did not point directly backward. Left-right inversion was associated with lower spatial abilities, and those six participants behaved as if they erroneously interpreted leftward optic flow as indicating leftward, not rightward motion and vice versa. Taken together, this study suggests that even an immersive high-quality video projection system is not necessarily sufficient for enabling natural spatial orientation in VR if no landmarks are provided.

Funding: EU grant POEMS-IST-2001-39223 and the Max Planck Society.

\section{References}

Klatzky, R. L., Loomis, J. M., Beall, A. C., Chance, S. S., And Golledge, R. G. 1998. Spatial updating of self-position and orientation during real, imagined, and virtual locomotion. Psychol. Sci. 9, 4, 293-298. 